\title{
Review of: "An atlas of human viruses provides new insights into diversity and tissue tropism of human viruses"
}

\author{
Evelyne Schvoerer ${ }^{1}$ \\ 1 Centre Hospitalier Régional Universitaire de Nancy (CHRU Nancy)
}

Potential competing interests: The author(s) declared that no potential competing interests exist.

\section{MANUSCRIPT}

Title: An atlas of human viruses provides new insights into diversity and tissue tropism of human viruses

Sifan Ye, Congyu Lu, Ye Qiu, Heping Zheng, Xingyi Ge, Aiping Wu, Zanxian Xia, Taijiao Jiang, Haizhen Zhu, Yousong Peng

Interest/Quality/Originality/Overall $=$ HIGH

Recommendation: REVISION

To summarize the work:

The manuscript has investigated human viruses from public databases and publications in order to propose a Human Virus Database (HVD), classified according to human tissues, excreta and body fluids. The main observations showed that the viral diversity varied according to patients' age and that the tissues were associated with several factors, i.e. DNA or RNA genome, enveloped or not, the length of viral genome and GC content, interacting viral receptors and virus-interacting proteins. The authors provided good Tables and figures showing their main results.

\section{Major comments:}

- First, as the authors suggested that the viral diversity varied according to patients' age, this should be deeply developed in the discussion.

Could the authors add an exploration of the publications $\mathrm{db}$, with the aim to analyze whether the teenagers might be protected from a high number of viral species or not.

- Second, the authors should discuss in detail how they hypothesized that the more receptors used by a virus, the larger the tissue range is, with a confirmatory tendency for DNA viruses. On the contrary, 1 unique but largely distributed receptor could be in favor of a larger tissue tropism?

Please could the authors develop this point in the Discussion. 


\section{Minor comments:}

The English language should be checked by an Englishborne subject.

\section{CONCLUSION for the editor}

The manuscript has investigated human viruses from databases and publications and discussed a Human Virus Database (HVD), exploring a highly interesting field of medical virology. But the paper should be improved as suggested in the major comments, and then re-submitted for publication as a revision procedure. 